\title{
Problemas na comunicação: uma possível causa de erros de medicação*
}

\author{
Communication problems: a potential cause for medication error-rates
}

Problemas en la comunicación: una posible causa de errores de medicación

\author{
Ana Elisa Bauer de Camargo Silva ${ }^{1}$, Silvia Helena de Bertoli Cassiani ${ }^{2}$, Adriana \\ Inocenti Miasso ${ }^{3}$, Simone Perufo Opitz ${ }^{4}$
}

\section{RESUMO}

Objetivo: Este estudo descritivo identificou e analisou pontos frágeis da comunicação durante a realização da prescrição, dispensação e administração de medicamentos na unidade de clínica médica e farmácia de um hospital universitário. Métodos: Os dados foram coletados através de observações diretas durante 21 dias; revisões de 294 prescrições de medicamentos e 40 entrevistas com profissionais. Resultados: A análise identificou prescrições incompletas, com abreviaturas, e realizadas sob interrupções e distrações. Na dispensação, as requisições apresentaram falhas no preenchimento dos formulários; na administração de medicamentos os problemas referiam-se ao uso de etiquetas transcritas para o preparo da medicação e falta de comunicação entre profissionais de enfermagem e paciente. Conclusão: Os meios de comunicação utilizados devem ser revistos, a fim de que se crie um sistema de medicação seguro para o paciente.

Descritores: Erros de medicação; Sistemas de medicação no hospital, Comunicação; Enfermagem; Hospitalização; Controle de risco

\begin{abstract}
Objective: This descriptive study identified and analyzed weak points in the communication process during the prescription, dispensing, and administration of medication in a medical unit and pharmacy of a university hospital. Methods: The data were collected by direct observations during a period of 21 days, review of 294 prescriptions, and interview of 40 health care professionals. Results: Some prescriptions were incomplete, common use of abbreviations, and health care providers were often interrupted or distracted during prescription. During the dispensing phase, many requisition forms were incomplete or filled out wrongly. And, during the administration of medication, there were errors on transcribed labels for the preparation of medication as well as a lack of proper communication between nurses and patients. Conclusion: Communication process in place must be revised to guarantee a quality hospital medication system that provides safe patient care.
\end{abstract}

Keywords: Medication errors; Medication systems, hospital, Communication, Nursing; Hospitalization; Risk management

\section{RESUMEN}

Objetivo: En este estudio descriptivo se identificó y analizó los puntos frágiles de la comunicación durante la realización de la prescripción, dispensación y administración de medicamentos en la unidad de clínica médica y farmacia de un hospital universitario. Métodos: Los datos fueron recolectados a través de observaciones directas durante 21 días; revisiones de 294 prescripciones de medicamentos y 40 entrevistas con profesionales. Resultados: El análisis permitió la identificación de prescripciones incompletas, con abreviaturas, y realizadas bajo interrupciones y distracciones. En la dispensación, las requisiciones presentaron fallas en el llenado de los formularios; en la administración de medicamentos los problemas se referían al uso de etiquetas transcritas para la preparación de la medicación y falta de comunicación entre enfermería y paciente. Conclusiones: Los medios de comunicación utilizados deben ser revisados, a fin de que se cree un sistema de medicación seguro para el paciente.

Descriptores: Errores de medicación; Sistemas de medicación en el hospital, Comunicación; Enfermería; Hospitalización; Control de riesgo

\footnotetext{
* Trabalho realizado na Unidade de Clínica Médica e na Farmácia de um Hospital Universitário de Goiás.

${ }^{1}$ Mestre, Professora Assistente da Faculdade de Enfermagem da Universidade Federal de Goiás- UFG- Goiania (GO), Brasil. Aluna do Programa Interunidades de Doutoramento da Escola de Enfermagem de Ribeirão Preto - USP.

${ }^{2}$ Doutora, Professora Titular do Departamento de Enfermagem Geral e Especializada da Escola de Enfermagem de Ribeirão Preto. Universidade de São Paulo- USP- Ribeirão Preto (SP), Brasil.

${ }^{3}$ Professora Assistente do Departamento de Enfermagem Psiquiátrica e Ciências Humanas da EERP-USP. Universidade de São Paulo- USP-Ribeirão Preto (SP), Brasil.

${ }^{4}$ Doutora, Professora Assistente da Universidade Federal do Acre- UFAC - Rio Branco (AC), Brasil.
} 


\section{INTRODUÇÃO}

Erros durante a assistência prestada à saúde da população ocorrem em cerca de $14 \%$ dos pacientes hospitalizados nos EUA, sendo que os erros de medicação foram responsáveis pela morte de, aproximadamente, 7.000 americanos e por cerca de 30\% dos danos ocorridos durante a hospitalização, no ano de $1997^{(1-2)}$.

O sistema em que se desenvolve a medicação é complexo, no qual atuam médicos, equipes da farmácia e de enfermagem, que executam funções interdependentes. Dessa forma, uma falha em um dos processos, inclusive no de comunicação, poderá interferir no conjunto de atividades desenvolvidas, trazendo transtornos tanto à equipe como ao paciente e configurarse em uma das causas de erros de medicação.

Nesse contexto, uma das exigências para uma assistência de qualidade e segura é que o sistema possua um canal de comunicação eficaz, permitindo às equipes transmitir e receber informações de forma clara e correta.

A comunicação é um processo no qual ocorre a emissão, recepção e a compreensão das mensagens, que podem ser verbais (linguagem escrita e falada) e nãoverbais. A comunicação envolve relações interpessoais e, freqüentemente, podem ocorrer problemas, dificuldades e restrições, de maneira que a mensagem enviada não é decodificada corretamente ${ }^{(3)}$.

As mensagens a respeito da terapia medicamentosa são transmitidas pela comunicação escrita ou falada. $\mathrm{Na}$ escrita, utilizam-se as prescrições de medicamentos e formulários de requisição enviados à farmácia; as faladas ocorrem nas relações interpessoais e profissionais.

Os problemas de comunicação que podem gerar atos inseguros pertencem a três categorias: falhas no sistema em que o canal de comunicação não existe, não está funcionando ou não é regularmente utilizado; fracasso na emissão das mensagens, quando o canal de comunicação existe, mas a informação não é transmitida; falhas na recepção, quando o canal de comunicação existe, a mensagem foi enviada de maneira correta, mas o receptor a interpretou de forma equivocada ou com atraso ${ }^{(4)}$.

O Food and Drug Administration avaliou, no período de 1993 a 1998, os relatórios de erros de medicamentos que foram fatais e identificou que 16\% das causas desses erros foram atribuídas à falha na comunicação ${ }^{(5)}$.

Segundo o Joint Commission on Acreditation of Healthcare Organizations, falhas na comunicação foram consideradas a causa de mais de $60 \%$ dos 2.966 eventos sentinela ocorridos entre os anos de 1995 e 2004, sendo que desses, $326(10,99 \%)$ foram atribuídos a erros de medicação $^{(6)}$.

Assim, o sistema de medicação das instituições de saúde requer um processo de comunicação eficaz, estruturado de maneira a promover condições que auxiliem os profissionais na prevenção dos erros, para assegurar que o tratamento medicamentoso seja cumprido de forma segura.

\section{OBJETIVO}

Este estudo teve como objetivo identificar e analisar os pontos frágeis da comunicação existentes nos processos do sistema de medicação.

\section{MÉTODOS}

Trata-se de um estudo descritivo, realizado na unidade de clínica médica e na farmácia de um hospital universitário, escolhido por ser integrante do projeto de Hospitais Sentinela da Agência Nacional de Vigilância Sanitária e por ser campo de estágio de centros formadores de profissionais de saúde.

Após aprovação do estudo no Comitê de Ética em Pesquisa do hospital e consentimento escrito dos profissionais, deu-se início à coleta dos dados, a qual foi realizada em três etapas. $\mathrm{Na}$ etapa inicial, foram realizadas observações diretas e não-participantes dos processos de prescrição, dispensação, distribuição e administração de medicamentos, durante 21 dias consecutivos. Para tanto, utilizou-se um roteiro estruturado com a finalidade de obter descrição das atividades inerentes a esses processos, viabilizando a identificação de problemas na comunicação. A segunda etapa constou de entrevistas semi-estruturadas com 12 médicos $(\mathrm{M})$; 20 profissionais de enfermagem, sendo 4 enfermeiros (E), 14 técnicos de enfermagem (TE) e 2 auxiliares de enfermagem (AE), 8 profissionais da farmácia sendo 3 farmacêuticos $(F)$ e 5 técnicos de farmácia (TF). Participaram dessa etapa os profissionais que atuavam na clínica médica e na farmácia hospitalar, no período da investigação, desempenhando funções relacionadas à medicação. $\mathrm{O}$ objetivo dessa etapa foi obter opiniões sobre os tipos e causas de erros de medicação mais freqüentes. Na última etapa, foi realizada análise de 294 prontuários dos pacientes internados na clínica médica, no período da coleta de dados, durante sete dias consecutivos. Utilizou-se, para tal análise, um roteiro estruturado com o objetivo de identificar inadequações nas prescrições médicas, assim como analisar as anotações de enfermagem.

Para análise dos dados foi utilizada a abordagem qualiquantitativa. Após digitação e categorização dos dados, estes foram analisados pelos pesquisadores que os apresentaram na forma de freqüencias absolutas e relativas e de depoimentos expressos pelos entrevistados.

\section{RESULTADOS}

No processo de prescrição de medicamentos, 
principal fonte de informação da terapêutica medicamentosa, pôde-se observar que aquela era realizada manualmente e em duas vias, com papel carbono, e que, das 294 prescrições analisadas, 102 (34,7\%) delas estavam ilegíveis, ou parcialmente legíveis.

Quanto às informações que devem constar nas prescrições de medicamentos, pôde-se verificar que 279 $(94,9 \%)$ estavam incompletas para um ou mais itens: 228 $(77,6 \%)$ sem a apresentação do medicamento; 111 $(37,8 \%)$ sem a forma de diluição; $63(21,4 \%)$ sem a dose; $21(7,1 \%)$ sem a via de administração e $16(5,4 \%)$ não especificavam a freqüência com que o medicamento deveria ser administrado. Outro fator negativo identificado foi a utilização de abreviaturas, presentes em $282(95,9 \%)$ prescrições.

Os resultados das entrevistas com os profissionais mostraram que, em $29 \%$ dos relatos, o erro de prescrição foi apontado como o de maior ocorrência. Para esses profissionais, tais erros ocorrem devido à dificuldade de leitura e à incompreensão da letra do médico. As frases abaixo ilustram essa situação.

... alguns médicos fazem uma caligrafia que não dá pra você ler. Você tem que olhar nas prescrições anteriores... Alguém que tem mais experiência tem que vir olhar, porque já conhece a letra ... (TE)

Os médicos não prescrevem de forma completa e não descrevem se a medicaşão é $V O$ ou IV, comprimido ou ampola, então a pessoa responsável pela distribuição do medicamento olha, não entende e envia o que vem na cabeça; é errado, mas acontece. (TF)

...várias vezes que eu estava de plantão a funcionária de enfermagem trażia a prescrição para perguntar para gente, e nem a gente entendia, e dai você tem que ligar para quem prescreveu para entender o que ela quer. (M)

No processo de dispensação e distribuição de medicamentos pôde-se observar a existência de problemas de comunicação entre as equipes de farmácia, de enfermagem e de médicos quanto à existência ou não de medicamentos no hospital ou na unidade de internação. Nos relatos abaixo, vê-se que a dificuldade na comunicação resulta em prejuízo para o paciente.

Às vezes, a medicação fica sem ser feita porque não tem no hospital. A gente prescreve os antibióticos porque acredita que tenha e tem época em que o hospital tem deficiência desses medicamentos. (M)

.... a medicação acabou no bospital e não foi comunicado ao médico plantonista para mudança na prescrição... (M)

A funcionária de enfermagem é obrigada a olhar, ver se tem o medicamento, mas ela não avisou que não tinha e o paciente ficou sem a medicação. (M)

Identificou-se, também, que a farmácia deixa de fornecer à unidade medicamentos como psicotrópicos e antibióticos, devido a falhas no preenchimento das requisições padronizadas ou à falta das mesmas, ficando o paciente sem receber a terapêutica medicamentosa prescrita, sofrendo, portanto, um erro de omissão do medicamento ou um erro de horário.

...não administração de medicamentos por falta de controle de antibiótico ou psicotrópico, ou medicamento que acabou no hospital e não foi comunicado ao médico... (M)

Muitas medicações aqui funcionam através de controles, e o residente vem e prescreve, mas não faz o controle. A farmácia não manda o medicamento de jeito nenbum, então o paciente fica sem o antibiótico (E).

Quanto ao processo de preparo e de administração de medicamentos, também se constataram problemas como: o preparo de medicamentos baseado em transcrições das prescrições em etiquetas nas quais não constavam os nomes dos pacientes, apenas o número do seu leito, dado insuficiente, pois com freqüência trocam-se os pacientes de leito e de enfermaria. Ressaltase, aqui, o fato observado de que, na clínica referida, um transcreve, outro prepara e o outro administra o medicamento, fazendo com que o processo de medicar sofra múltiplas transferências de informações, o que pode gerar erros de medicação.

Outro problema observado foi o fato de a enfermagem administrar o medicamento sem falar com o paciente, chamando-o, no máximo, pelo nome.

Em relação às anotações de enfermagem, a análise das prescrições indicaram que apenas $45,4 \%$ dos medicamentos não administrados apresentavam justificativas, sendo estas reduzidas a anotações simples como "não tem" e "falta na farmácia", além do círculo em torno do horário aprazado.

\section{DISCUSSÃO}

No processo de prescrição de medicamentos, a utilização do papel carbono foi vista como um fator positivo, pois elimina a fase de transcrição que seria um passo adicional para se incorrer em erro; no entanto, quando de má qualidade, a segunda via da prescrição não apresentará boa resolução, podendo ocasionar problemas relacionados à sua legibilidade, com conseqüente erro no processo seguinte.

A esse respeito, o National Coordinating Council for Medication Error Reporting and Prevention (NCCMERP) enfatiza que a ilegibilidade das prescrições de medicamentos pode levar o profissional a interpretar de maneira errada a terapêutica desejada e causar danos aos pacientes ${ }^{(7)}$. Assim, é compromisso ético do profissional de medicina fazer uma prescrição completa, 
com caligrafia legível e informações claras e compreensíveis por toda a equipe.

Em estudo realizado no Brasil, 43,8\% dos profissionais de enfermagem apontaram a caligrafia do médico, ilegível ou difícil de ser lida, como uma das quatro causas mais freqüentes para a ocorrência de erros de medicação ${ }^{(8)}$.

Quanto às abreviaturas, sabe-se que essas economizam o tempo do prescritor, porém, quando mal empregadas, podem causar equívocos de leitura ou de interpretação, pois uma abreviatura pode ter mais de um significado, além de aumentar o trabalho daqueles que tentam compreendê-la ${ }^{(9)}$.

O NCCMERP faz algumas recomendações para a elaboração de prescrições legíveis: utilizar o sistema computadorizado; evitar abreviaturas; incluir nome, registro, idade e peso do paciente; inclusão da especialidade farmacêutica, dose, forma, diluição, concentração, quantidade, freqüência de administração e via de administração; duração da terapia, nome e assinatura do médico, assim como número de seu registro no conselho profissional ${ }^{(7)}$.

A maioria dos prescritores não cumpre as diretrizes técnicas e legais exigidas, por esse motivo suas prescrições tornam-se potenciais para erros em processos subseqüentes do sistema ${ }^{(10)}$.

Quanto aos problemas identificados no processo de dispensação e distribuição de medicamentos, acreditase que a aquisição de máquinas leitoras de códigos de barra, gerando relatórios de controle de estoques e dispensação na farmácia ou na administração dos medicamentos, são estratégias eficazes para auxiliar na divulgação de informações fidedignas e atualizadas para a equipe sobre os medicamentos disponíveis no hospital $^{(5,10)}$.

Ao profissional de enfermagem caberá, além de comunicar verbalmente a falta de algum medicamento, na unidade, à equipe médica e à farmácia, preocupar-se em fazer anotações no prontuário. Deve também justificar o motivo da não-administração do medicamento, pois isso o respaldará pela omissão daquela dose, e ainda sinalizará, para a equipe, a falta do medicamento, a recusa do paciente ou outra razão da não-administração do medicamento ${ }^{(11)}$.

Essas anotações devem ir além de um círculo em torno do horário da medicação, símbolo esse, utilizado pela enfermagem, que não pode ser considerado uma comunicação efetiva para todos os elementos da equipe.

A utilização da prescrição computadorizada de medicamentos como antibióticos e psicotrópicos também é uma solução indicada para o problema de preenchimento de requisições obrigatórias ${ }^{(9)}$.

Quanto ao processo de preparo e administração de medicamentos, estratégias devem ser implementadas para assegurar que o mesmo seja embasado na leitura das prescrições, eliminando etapas intermediárias como a transcrição em etiquetas. Nas identificações dos medicamentos preparados, deve-se garantir a existência de dados como nome e leito dos pacientes, além de pulseiras de identificação que devem existir para os pacientes $^{(12)}$.

O hábito de chamar o paciente pelo nome não substitui a necessidade de assegurar a identificação do mesmo através da pulseira. O uso do código de barras nas pulseiras e do "scanner" (para leitura do mesmo), antes da administração do medicamento, constitui outra forma apropriada de assegurar que o medicamento prescrito está sendo administrado ao paciente correto. Estima-se a redução de cerca de 70\% nas taxas de erros em uma instituição que utiliza um sistema de código de barras. Entretanto, os custos imediatos envolvidos nessa tecnologia podem ser altos ${ }^{(12)}$.

Sabe-se que a equipe de enfermagem é capaz de interceptar até $86 \%$ dos erros na medicação provindos dos processos de prescrição, transcrição e de dispensação, ao passo que apenas $2 \%$ dos erros na administração são interceptados ${ }^{(13)}$. Desse modo, o paciente constitui a última barreira para evitar-se um erro de medicação. Ele tem o direito de ser informado sobre todos os aspectos de sua terapêutica medicamentosa, tendo, assim, a oportunidade de, por meio do conhecimento e de uma comunicação efetiva, tornar-se participante ativo no seu próprio tratamento.

Quanto às anotações de enfermagem, sabe-se que esses registros são fontes importantes de informações e devem ser valorizados, pois são utilizados para fins administrativos, podendo refletir a qualidade do cuidado prestado assim como a preocupação com uma comunicação eficiente entre os membros da equipe de saúde.

\section{CONCLUSÃO}

Diante do exposto, este estudo identificou pontos frágeis na comunicação em cada um dos processos do sistema de medicação analisado, que podem levar os profissionais a desenvolver atos inseguros como: prescrições de medicamentos incompletas e com abreviaturas não padronizadas que levam à incompreensão das mensagens enviadas; falhas no preenchimento de requisições e formulários que levam ao não fornecimento de medicamentos ao paciente; falhas no fornecimento de informações entre as equipes dos diferentes processos; uso de etiquetas de identificação dos medicamentos incompletas, assim como falta de comunicação entre as equipes e os pacientes, fazendo com que informações relevantes deixem de ser transmitidas. 
Os problemas de comunicação acarretam transtornos nas atividades da equipe multidisciplinar, a ponto de os profissionais culparem-se uns aos outros pelas falhas, fatos que ocasionam desgastes emocionais, atrasos e/ou omissão na administração dos medicamentos e ainda geram gastos desnecessários às instituições hospitalares. O indicado é que os processos de medicação sejam revistos, ações próativas e estratégias sejam implementadas, visando à melhoria da comunicação e garantia de uma terapêutica medicamentosa eficiente e segura aos pacientes. Dentre as possíveis estratégias sugerimos:

- confecção, padronização e divulgação de processos, procedimentos e de protocolos.

- implantação da prescrição eletrônica como forma de modernizar e tornar o sistema de medicação mais seguro ao garantir comunicação rápida e efetiva de informações entre elementos da equipe de saúde;

- identificação do paciente com pulseiras ou braceletes. Enquanto os equipamentos para a utilização do código de barras podem ter um custo elevado, o emprego das pulseiras de identificação e a apropriada identificação no leito geram menor custo e, se utilizadas efetivamente, contribuem para minimizar a ocorrência de administração de medicamentos a pacientes errados;

- incentivo do uso de pulseiras coloridas como uma forma de comunicar sobre alergias medicamentosas já conhecidas;

- estabelecimento de efetiva comunicação com o paciente, estimulando-o a questionar os vários aspectos da terapêutica medicamentosa, atuando como parceiro no tratamento, devendo ser conscientizado de que tem direito à informação sobre seu transtorno e sobre os medicamentos prescritos. O uso de apostilas, livretos e/ ou folhetos com explicações simples, que possam ser consultados sempre que necessário, pode constituir estratégia viável para o alcance desse objetivo;

- garantia da participação de um farmacêutico clínico na equipe. Esse pode ser diferencial importante para a orientação sobre o uso correto dos medicamentos e para o esclarecimento das dúvidas referentes ao tratamento;

- promoção da educação permanente, de forma a garantir a comunicação entre os membros da equipe de saúde e a deslocar o foco de uma equipe multiprofissional para o desenvolvimento de conhecimentos de caráter interdisciplinar.

\section{REFERÊNCIAS}

1. Bates DW, Miller EB, Cullen DJ, Burdick L, Williams L, Laird N, et al. Patient risk factors for adverse drug events in hospitalized patients. ADE Prevention Study Group. Arch Inter Med. 1999; 159(21):2553-60. Comment in: Arch Intern Med. 2000; 160(11):1700-1.

2. Kohn LT, Corrigan JM, Donaldson MS, editors. To err is human: building a safer health system. Washington, D.C.: National Academy Press; 2000.

3. Stefanelli MC. Comunicação com o paciente: teoria e ensino. 2a ed. São Paulo: Robe; 1993.

4. Reason J. Managing the risks of organizational accidents. Aldershot: Ashgate; 1997

5. Meadows M. Strategies to reduce medication errors. How the FDA is working to improve medication safety and what you can do to help. FDA Consum [serial on the Internet]. 2003 [cited 2003 Nov 16]; 37(3): [cerca de 7p.]. Available from: http://www.fda.gov/fdac/features/2003/303_meds.html

6. Joint Commission on Accreditation of Healthcare Organizations. Sentinel Event Statistics. 2005. [cited 2006 Dec 25]. Available from: http://www.jointcommission.org/SentinelEvents/Statistics/

7. National Coordinating Council for Medication Error Reporting and Prevention. Council Recommendation. Recommendations to Enhance Accuracy of Prescription Writing. [text on the Internet] 1996 [cited 2006 Dec 1]. NCC MERP. Available from: http:/ / www.nccmerp.org/council/ council1996-09-04.html

8. Bohomol E, Ramos LH. Erros de medicação: causas e fatores desencadeantes sob a ótica da equipe de enfermagem. Acta Paul Enfermagem. 2003; 16(2): 41-8.

9. Cohen MR. Preventing medications errors related to prescribing. In: Cohen MR. Medication errors causes, prevention, and risk management. Washington: American Pharmaceutical Association; 2000.

10. Lee P. Ideal principles and characteristics of a fail-safe medication-use system. Am J Health Syst Pharm. 2002; 59(4): 369-71. Erratum in: Am J Health Syst Pharm. 2002; 59(6):518.

11. Oliveira RC, Camargo AEB, Cassiani SHB. Estratégias para prevenção de erros na medicação no setor de emergência. Rev Bras Enfermagem. 2005; 58(4):399-404.

12. Miasso AI, Cassiani SHB. Erros na administração de medicamentos: divulgação de conhecimentos e identificação do paciente como aspectos relevantes. Rev Esc Enfermagem USP. 2000; 34(1):16-25.

13. Leape LL, Bates DW, Cullen DJ, Cooper J, Demonaco HJ, Gallivan T, et al. Systems analysis of adverse drug events. JAMA. 1995; 274(1): 35-43. 